\title{
Generalized Contour Dynamics: a review
}

\author{
Stefan G. Llewellyn Smith ${ }^{1,2 *}$, Ching Chang ${ }^{1 * *}$, Tianyi Chu ${ }^{1 * * *}$, \\ Mark Blyth ${ }^{3 * * * *}$, Yuji Hattori ${ }^{4 * * * *}$, and Hayder Salman ${ }^{3 * * * * *}$ \\ ${ }^{1}$ Department of Mechanical and Aerospace Engineering, Jacobs School of Engineering, UCSD \\ 9500 Gilman Drive, La Jolla CA 92093-0411, USA \\ ${ }^{2}$ Scripps Institution of Oceanography, UCSD \\ 9500 Gilman Drive, La Jolla CA 92093-0213, USA \\ ${ }^{3}$ School of Mathematics, University of East Anglia \\ Norwich, NR4 7TJ, UK \\ ${ }^{4}$ Institute of Fluid Science, Tohoku University \\ 2-1-1 Katahira, Aoba, Sendai Japan 980-8577 \\ Received TBD 00, 2018; accepted Month XX, 20XX
}

\begin{abstract}
-
Contour dynamics is a computational technique to solve for the motion of vortices in incompressible inviscid flow. It is a Lagrangian technique in which the motion of contours is followed, and the velocity field moving the contours can be computed as integrals along the contours. Its best-known examples are in two dimensions, for which the vorticity between contours is taken to be constant and the vortices are vortex patches, and in axisymmetric flow for which the vorticity varies linearly with distance from the axis of symmetry. This review discusses generalizations that incorporate additional physics, in particular buoyancy effects and magnetic fields that take specific forms inside the vortices and preserve the contour dynamics structure. The extra physics can lead to time-dependent vortex sheets on the boundaries, whose evolution must be computed as part of the problem. The non-Boussinesq case, in which density differences can be important, leads to a coupled system for the evolution of both mean interfacial velocity and vortex sheet strength. Helical geometry is also discussed, in which two quantities are materially conserved and whose evolution governs the flow.
\end{abstract}

Keywords: Vortex dynamics, contour dynamics, vortex patch, vortex sheet, helical geometry

\section{INTRODUCTION}

Vortex dynamics forms an important part of fluid dynamics, with a history dating back to the original paper of Helmholtz [13]. The search for exact solutions to the equations of motion has been a major part of the evolution of the field. Given the complexity of the nonlinear equations of motion, simplifications have been an important part of these efforts. Three general types of simplifications have been pursued: geometric, i.e. working in two-dimensional or axisymmetric geometries; model reduction, i.e. reducing partial differential equations to ordinary differential equations by considering singular solutions; and physical, i.e. limiting the physical effects considered. The motion of point vortices, one of the most important cases of reduced models, is reviewed in $[3,16]$. Point vortices are two-dimensional solutions for which the vorticity is given by a delta

\footnotetext{
*E-mail: sgls@ucsd.edu

** E-mail: chc054@ucsd.edu

***E-mail: tic173@ucsd.edu

${ }^{* * * *}$ E-mail: M.Blyth@uea.ac.uk

${ }^{* * * * *}$ E-mail: hattori@fmail.ifs.tohoku.ac.jp

******E-mail: H.Salman@uea.ac.uk
} 
function centered at a set of points whose locations evolve following a self-consistent set of dynamical equations.

Another important reduced model is that of vortex patches. These are two-dimensional solutions with piecewise constant vorticity, or equivalently a sum of Heaviside functions. Their evolution can be reduced to the evolution of the boundary, and this approach is called contour dynamics (CD) [31]. Good reviews are given in [9, 23]. A similar approach for axisymmetric flows was developed in [32] (see also [26]) and in [22]. Equilibrium solutions were first found in [29] (including rotating solutions generalizing the Kirchhoff ellipse) and [21] in the two-dimensional and axisymmetric cases. Contour dynamics allows simulations that are effectively inviscid with very low numerical diffusion or dissipation. It is found that the boundaries of vortex patches can filament, and techniques like contour surgery [9] can be used to pursue integrations. A major advantage over other numerical techniques is in efficiency, since it reduces the dimensionality of the system by one (in this regard it is similar to Boundary Element Methods for very viscous Stokes flows). Conversely, it is limited by the very fact that it cannot simulate viscous or three-dimensional flows. One goal of the present review is to outline how extra physics can be included in the method.

Yet another model is that of vortex sheets, in which the vorticity is made of delta functions located along curves or surfaces. The resulting velocity field is discontinuous across the sheets. Again dynamical equations of motion result, which are well-known to be ill-posed: vortex sheets are susceptible to Kelvin-Helmholtz instability. Regularization methods [14] exist to produce wellposed equation sets.

These reduced models are reviewed in textbooks on vortex dynamics such as $[1,4,6]$. Contour dynamics specifically is a broad field; rather than provide an exhaustive bibliography, we refer the reader to $[4,9,23]$ for detailed discussions and to [20] for a comprehensive bibliography of vortex dynamics as a whole. Our goal in this review article is to present extensions to contour dynamics that incorporate more physics in various geometric configurations.

We define generalized contour dynamics (GCD) methods as solutions to the Helmholtz vorticity equations that are fully determined by the evolution of contours or surfaces. In standard contour dynamics, these contours are vortex jumps, but including additional physics leads to the creation and evolution of vortex sheets. In such generalisations, two-dimensional, axisymmetric or helical symmetry are needed to ensure material conservation of vorticity or its close relatives, while the magnetic field is perpendicular to the contours.

In this review we include density differences, which in combination with gravity lead to buoyant effects, and magnetic fields. It is immediately clear that density differences will lead to baroclinic generation of vorticity, which will affect the dynamics. Magnetic fields have previously been considered in [12]. We do not review numerical methods to solve the resulting sets of equations, nor the question of finding equilibrium solutions. Similarly, while equilibrium solutions can be obtained for spherically symmetric solutions, with and without swirl [17], since their boundary is steady they do not really fall within the scope of this review.

Most of the results in this review are already known, but putting them together highlights the differences and similarities between the different cases. The axisymmetric non-Boussinesq result appears to be new, although it is formally the same as the 2D result (up to the relation between vortex sheet strength and circulation; see below). The equations for the helical case appear new but their existence had been suggested earlier; see [23].

In $\S 2$ we review the equations of motion and outline the underlying physical and geometrical assumptions that will be used. In $\S 3$ we describe a vorticity-based approach and obtain results that include effects due to buoyancy and magnetic field. In $\S 4$ we describe the modifications needed in the non-Boussinesq case, and include the effect of surface tension. In $\S 5$ we discuss the case of helical symmetry. Finally we conclude in $§ 6$.

\section{GOVERNING EQUATIONS}

\subsection{Field Equations}

We will define a generalized contour dynamics method as one in which vorticity-like quantities

are conserved materially, so that the evolution of the system is governed by the motion of boundaries between different regions. In practice this means two-dimensional, axisymmetric or helical geometries to avoid vortex stretching terms that break the conservation of vorticity-like 
quantities. Three features are required: a Lagrangian conservation law, an inversion integral or operator (via a Green's function), and a vortex sheet evolution equation that takes into account additional physics. Vorticity-like quantities are taken to be piecewise continuous. Vorticity, axial velocity and magnetic field are non-zero inside a vortical region and vanish outside. The other velocity components are non-zero over the whole of space.

We start with the general vorticity equation for three-dimensional inviscid incompressible flow in the presence of magnetic field. This requires the induction equation, in which we also assume infinite magnetic Reynolds number. We have

$$
\begin{aligned}
\frac{\mathrm{Du}}{\mathrm{D} t} & =-{ }^{\frac{1}{\rho}} \nabla p+\mathbf{g}+\mathbf{f}+\frac{\rho_{0}}{\rho} \mathbf{j} \times \mathbf{B}, \\
\nabla \cdot \mathbf{u} & =0, \\
\mathrm{D} \mathbf{B} & =\mathbf{B} \cdot \nabla \mathbf{u}, \\
\overline{\mathrm{D} t} & =0, \\
\nabla \cdot \mathbf{B} & =0, \\
\frac{\mathrm{D} \rho}{\mathrm{D} t} & =0 .
\end{aligned}
$$

where $\mathbf{u}$ is the velocity field, $p$ is the pressure field, $\rho$ is the density field, $\mathbf{g}$ is the gravitational acceleration, taken to be constant, and $\mathbf{f}$ stands for the remaining body force (per unit mass). We require the total body force to be time-independent and conservative so that $\mathbf{g}+\mathbf{f}-\nabla \Psi$. The current density is given by $\mathbf{j}=\nabla \times \mathbf{B}$, where $\mathbf{B}$ is the magnetic field, and units have been chosen to obtain the simplest possible form for the equations, with $\rho_{0}$ a constant reference density. Density is materially conserved, i.e. diffusion of density is neglected. The vorticity equation for $\boldsymbol{\omega}$

$$
\underline{\mathrm{D} \boldsymbol{\omega}}=\frac{1}{\rho^{2}} \nabla \rho \times \nabla p+\nabla \times \underset{\rho}{\rho_{0}}(\mathbf{j} \times \mathbf{B}),
$$

with $\boldsymbol{\omega}$ the vorticity, which does not look immediately promising for Lagrangian conservation.

To simplify matters initially, we introduce the Boussinesq approximation, which corresponds to neglecting changes in density except in the gravitational term [5]. We will set $\mathbf{g}=g \mathbf{l}$ where $\mathbf{l}$ is a unit vector pointing in the opposite direction to gravity and define the buoyancy, $b$, by

$$
\rho=\rho_{0}\left(1-g^{-1} b\right) \text {. }
$$

(The usual situation is $\mathbf{l}=\hat{\mathbf{z}}$, but we use $\mathbf{l}$ to accommodate the two-dimensional case with flow and gravity in the $x y$-plane so that $\mathbf{g}$ does not point in the negative $z$-direction.) The resulting momentum, continuity and buoyancy equations are

$$
\frac{\mathrm{Du}}{\mathrm{D} t}=-\frac{1}{\rho_{0}} \nabla p+b \mathbf{l}+\mathbf{f}+\mathbf{j} \times \mathbf{B}, \quad \boldsymbol{\nabla} \cdot \mathbf{u}=0, \quad \frac{\mathrm{D} b}{\mathrm{D} t}=0,
$$

where the pressure has had its hydrostatic component removed. The vorticity equation simplifies to

$$
\frac{\mathrm{D} \boldsymbol{\omega}}{\mathrm{D} t}=\nabla b \times \mathbf{l}+\nabla \times(\mathbf{j} \times \mathbf{B}) .
$$

Contour dynamics is a Lagrangian approach. Points on the boundary $\mathbf{X}(\xi, t)$ are advected as Lagrangian markers:

$$
\frac{\partial \mathbf{X}}{\partial t}=\mathbf{u}(\mathbf{X})
$$

where $\xi$ is any parameterization of the boundary (not necessarily arc length). Numerically, the contours are discretized into points $\left\{\mathbf{X}_{k}\right\}$ and the points are advanced according to the coupled ordinary differential equations

$$
\frac{\mathrm{d} \mathbf{X}_{k}}{\mathrm{~d} t}=\mathbf{u}\left(\mathbf{X}_{k}\right)
$$


Hence the velocity field on the boundary is needed. Note that while material points move with the velocity field, the boundary itself is a geometric entity.

In three dimensions, total energy, helicity and cross helicity are conserved. The general form for these quantities is

$$
E_{T}=\frac{1}{2}\left(\rho|\mathbf{u}|^{2}+\rho \Psi+\left.\rho \oint \mathbf{B}\right|^{2}\right) d V, \quad H=\boldsymbol{u} \cdot \boldsymbol{\omega} d V, \quad H_{C}=\boldsymbol{u} \cdot \mathbf{B} d V
$$

respectively. Helicity vanishes in the bulk unless the swirl velocity is non-zero; similarly cross helicity is zero unless both swirl and toroidal field are non-zero. Magnetic helicity is also preserved, but the assumptions made here imply that it vanishes. (In two dimensions, helicity conservation is replaced by conversation of enstrophy and moments of vorticity.) In general, vortices with different buoyancy from the outside fluid will keep moving parallel to the gravity vector and will continuously change shape [34], and no equilibrium solutions are likely in this case.

We now consider possible geometries for contour dynamics. This includes the relation between vorticity and (part of the) velocity, a kinematic result that does not depend on the momentum or vorticity equations. Helical geometry is more complicated and is discussed separately in $\S 5$.

\section{2. $2 \frac{1}{2}$-Dimensional Flows}

The usual two-dimensional configuration has $\mathbf{u}=(u(x, y, t), v(x, y, t))$ and $\boldsymbol{\omega}=\omega(x, y, t) \hat{\mathbf{z}}$. Since the flow is incompressible, a streamfunction $\psi$ exists with $(u, v)=\left(\psi_{y},-\psi_{x}\right)$. It is possible to add a third velocity component, and consider a $2 \frac{1}{2}$-dimensional velocity field $[18,24]$ when the velocity components and pressure gradient do not depend on $z$, so that $\mathbf{u}=(u(x, y, t), v(x, y, t), w(x, y, t))$. The vertical velocity then behaves as a passive scalar. To obtain a CD system, we need $\omega, w$ and density $\left(\rho\right.$ or $b$ ) to be piecewise continuous. We have already required $\mathbf{f}=\left(f_{x}(x, y, t), f_{y}(x, y, t), 0\right)$ to be irrotational. We also need the $z$-component of $\mathbf{g}$ to vanish. The induction equation can be written in a $\mathrm{CD}$ form if $\mathbf{B}=(0,0, B(x, y, t))$. Then $B, w$ and $\omega$ are materially conserved in the bulk.

The Poisson equation $\nabla^{2} \psi=-\omega$ can be solved using a Green's function approach, giving

$$
\psi=-\frac{1}{2 \pi}^{\mathbf{J}} \omega\left(\mathbf{x}^{j}\right) \log \left|\mathbf{x}-\mathbf{x}^{j}\right| d \mathbf{x}^{j}
$$

We are taking $\omega$ to be a sum of vortex patches and vortex sheets, corresponding to Heaviside and delta functions respectively. The latter is naturally a contour integral, while the former can be turned into one for a patch of constant vorticity $A$. We write $\mathbf{u}_{H}$ for the velocity induced by the patch and $\mathbf{u}_{S}$ for the velocity induced by the sheet. The resulting contour integrals can be written in the form

$$
\mathbf{u}_{H}=-\underline{A}_{2 \pi}^{\mathbf{I}} \log \left|\mathbf{x}-\mathbf{x}^{j \mid}\right|_{\frac{d \mathbf{x}^{j}}{\partial s^{j}}}^{d s^{j},} \quad \mathbf{u}_{S}=\hat{\mathbf{z}} \times \frac{1}{2 \pi}^{\mathbf{I}} \gamma \frac{\mathbf{x}-\mathbf{x}^{j}}{\left|\mathbf{x}-\mathbf{x}^{j}\right|^{2}} d s^{j},
$$

where $s^{j}$ represents arc length along the contour which is described in the positive sense and $\gamma(s, t)$ is the vortex sheet strength (the jump in velocity across the contour). In the above we are ignoring external velocity fields and boundaries. The total velocity in the $x y$-plane is $\left(\psi_{y},-\psi_{x}\right)=\mathbf{u}_{H}+\mathbf{u}_{S}$.

The energy and cross helicity can be written as

$$
E_{T}=\frac{1}{2}_{V}^{\mathbf{J}}\left(\rho w^{2}+\rho|\nabla \psi|^{2}+\rho \Psi+\rho_{0} B^{2}\right) d V, \quad H_{C}={ }_{V}^{\mathrm{J}} w B d V,
$$

where $V$ is the inside of the vortex. In the Boussinesq limit, we replace $\rho$ by $\rho_{0}$ in the energy integral; then $\rho_{0}$ can be taken out of the integral. 


\subsection{Axisymmetric Flows}

We use cylindrical coordinates $(r, \theta, z)$ with velocity components $(u, v, w)$. Then, for incompressible axisymmetric flow with variables depending on $r, z$ and $t$, the radial and vertical velocity components are related to the Stokes streamfunction $\psi$ by

$$
u=-\frac{1}{r} \frac{\partial \psi}{\partial z} \quad w=\frac{1}{r} \frac{\partial \psi}{\partial r} .
$$

The relation between streamfunction and azimuthal vorticity $\omega$ is

$$
\omega=\frac{\partial u}{\partial z}-\frac{\partial w}{\partial r}=-\frac{1}{r} \frac{\partial^{2}}{\partial r^{2}}-\frac{1 \partial}{r \partial r}+\frac{\partial^{2}}{\partial z^{2}} \psi
$$

The swirl velocity $v$ is separate [17]. To obtain a CD system, we require gravity to point in the negative $z$-direction (i.e. $\mathbf{l}=\hat{\mathbf{z}}$ ) and the magnetic field to be purely azimuthal (also called toroidal) [12].

The relation (2.17) can be inverted to give

$$
\psi(r, z, t)=\int \omega\left(r^{j}, z^{j}, t\right) t t\left(r, z \mid r^{j}, z^{j}\right) d r^{j} d z^{j},
$$

where $t t$ is the Green's function

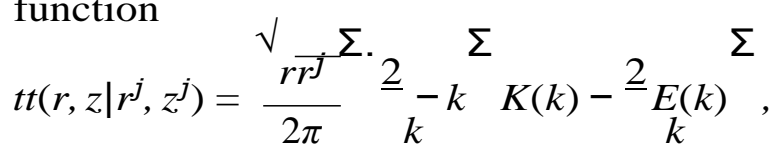

$K(k)$ and $E(k)$ are the complete elliptic integrals of the first and second kind, respectively, and

$$
k^{2}=\frac{4 r r^{j}}{\left(r+r^{j}\right)^{2}+\left(z-z^{j}\right)^{2}} .
$$

When $\omega=r A$ inside a contour, $A$ constant, and vanishes outside, the velocity field can be rewritten in terms of contour integrals described in the clockwise sense [12, 25, 26]. Again we write $\mathbf{u}_{H}$ for the velocity induced by the patch and $\mathbf{u}_{S}$ for the velocity induced by the sheet, so that

$$
u_{H}(r, z, t)=-\underline{A}_{r} t t \cos \theta d s^{j}, \quad w_{H}(r, z, t)=A \quad\left[\left(z^{j}-z\right) H \cos \theta-t t \sin \theta\right] d s^{j}
$$

for the velocity components. Here $\theta$ is the angle between the outward normal to the interface and the $z$-axis, while $H$ is given by

$$
H(r, z \mid r, z)=\frac{r^{j} K(k)}{\pi \quad \frac{\left(r+r^{j}\right)^{2}+\left(z-z^{j}\right)^{2}}{}}
$$

For later use, we require the velocity field induced by a vortex sheet with strength $\gamma$

$$
u_{S}(r, z, t)=-\frac{1}{r} \gamma \frac{\partial t t}{\partial z} d s^{j}, \quad w_{S}(r, z, t)=\frac{1}{r} \gamma \frac{\partial t t}{\partial r} d s^{j} .
$$

The total poloidal velocity is $\mathbf{u}_{P}=\mathbf{u}_{H}+\mathbf{u}_{S}$, with $\left|\mathbf{u}_{P}\right|^{2}=|\nabla \psi|^{2}$, while the toroidal velocity is $v$.

The energy and cross helicity can be written as

$$
E_{T}=\frac{1}{2}{ }_{V} \rho v^{2}+\frac{1}{r^{2}} \rho|\nabla \psi|^{2}+\rho \Psi+\rho B^{2} d V, \quad H_{C}={ }^{\delta} w d V .
$$

In the Boussinesq limit, we replace $\rho$ by $\rho_{0}$ in the energy integral; then $\rho_{0}$ can be taken out of the integral. 


\section{VORTICITY-BASED APPROACH FOR BOUSSINESQ FLOWS}

\subsection{Axisymmetric Case}

We now derive contour dynamics equations following $[12,17]$. This approach exploits the Boussinesq approximation to avoid dealing with the baroclinic torque terms in (2.6). We derive the axisymmetric version, since its simplification to two dimensions is immediate. We consider only a single contour, but the generalization to more than one is straightforward.

From the form of the solution in $\$ .3$, the prognostic equations are the azimuthal momentum, vorticity and magnetic induction equations, which become

$$
\frac{\mathrm{D}}{\mathrm{D} t}(r v)=0, \quad \frac{\mathrm{D}}{\mathrm{D} t} \frac{\underline{\omega}}{r}^{\Sigma}=\frac{1 \partial}{r^{2} \partial z}\left(v^{2}-B^{2}\right)-\frac{1 \partial b}{r \partial r}, \quad \frac{\underline{\mathrm{D}}^{-}}{\mathrm{D} t} \underline{B}^{\Sigma}=0 .
$$

Take the azimuthal vorticity in the form

$$
\omega=r[A H(f)+\Omega|\nabla f f| \delta(f)]
$$

where $H$ is the Heaviside step function, $\delta$ is the Dirac delta function and $\Omega$ is the vortex sheet strength. The function $f(r, z, t)$ is an indicator function that is negative inside the vortex and positive outside. Similarly we take the buoyancy, swirl and magnetic field in the form

$$
b=b_{0} H(f), \quad r v=C H(f), \quad B=r K H(f)
$$

for constant $b_{0}, C$ and $K$. Then the cross helicity is given by $H_{C}=K C V$, where $V$ is the volume of the vortex.

The vortex sheet strength $\Omega$ evolves in time, while $A, b_{0}, C_{\Sigma}$ and $K$ are constant. We find

$$
\frac{\mathrm{D} \Omega}{\mathrm{D} t}+\frac{\Omega \mathrm{D} \mid \boldsymbol{\nabla} \boldsymbol{f l}}{|\boldsymbol{\nabla} \boldsymbol{f}| \mathrm{D} t}=\frac{1}{|\boldsymbol{\nabla} \boldsymbol{f}|} \frac{C^{2}}{r^{4}}-K^{2} \frac{\partial \boldsymbol{f}}{\partial z}-\frac{b_{0} \partial \boldsymbol{f}}{|\boldsymbol{\nabla} \boldsymbol{f}| \partial \boldsymbol{r}}
$$

The function $f$ is materially conserved. We take the boundary of the vortex to be $(r, z)=$ $(R(\xi, t), Z(\xi, t))$. Then the results

$$
L^{2}(\xi, t)=-\frac{\partial R^{\Sigma_{2}}}{\partial \xi}+{ }^{-} \frac{\partial Z^{\Sigma_{2}}}{\partial \xi}, \quad \mathbf{n}=\frac{\boldsymbol{\nabla} f}{|\boldsymbol{\nabla} f|}, \quad \frac{1 \mathrm{D}|\boldsymbol{\nabla} f|}{|\boldsymbol{\nabla} f| \mathrm{D} t}=\frac{1 \partial L}{L \partial t}+\frac{u}{r},
$$

which in particular define $L$, can be used to obtain a simple dynamical equation for $\gamma=\Omega L R$, where $\mathrm{d} \gamma$ is the circulation in a small area which includes a portion $\mathrm{d} s$ of the boundary [12]:

$$
\frac{\partial \gamma}{\partial t}=K^{2} R-\frac{C^{2}}{R^{3}} \frac{\partial R}{\partial \xi}-b_{0} \frac{\partial Z}{\partial \xi}
$$

This is a generalization of the results of $[11,12,17]$ to include swirl, magnetic field and buoyancy.

\subsection{Two-dimensional Case}

Without loss of generality take $\mathbf{l}$ to point in the $y$-direction (see the discussion in $\S 2$ ). The governing equations simplify to

$$
\frac{\mathrm{D} w}{\mathrm{D} t}=0, \quad \frac{\mathrm{D} \omega}{\mathrm{D} t}=-\frac{\partial b}{\partial x^{\prime}} \quad \frac{\mathrm{D} B}{\mathrm{D} t}=0 .
$$

We see that magnetic field and vertical velocity have no dynamical impact on the evolution of $\omega$. If we set

$$
\omega=A H(f)+\Omega|\nabla \boldsymbol{f}| \delta(\boldsymbol{f}), \quad b=b_{0} H(f), \quad w=C H(f), \quad B=K H(f),
$$

the relation between $\Omega$ and $\gamma$ is now $\gamma=\Omega L$. The evolution equation for $\gamma$ becomes

$$
\frac{\partial \gamma}{\partial t}=-b_{0} \frac{\partial Y}{\partial \xi}
$$

where the contour is now given by $(x, y)=(X(\xi, t), Y(\xi, t))$. 


\section{NON-BOUSSINESQ EFFECTS}

The vorticity approach above seems thwarted in the non-Boussinesq case because of the baroclinic torque term in (2.6). It is still true that the (appropriately modified) vorticity and buoyancy are materially conserved, so a CD formulation works, but the evolution equation for the vortex sheet needs to be obtained in a different manner. This has been done in the irrotational case by several authors. The case for different densities has been derived a number of times. [28] gives references and outlines a general approach, which says nothing about the fluid away from the interface.

Once again we start with the axisymmetric case. Our approach is similar to that of [7], but we do not assume irrotational flow. The unit tangent and outward normal to the contour $\mathbf{X}=(R, Z)$ parameterized by $\xi$ are

$$
\mathbf{t}=\frac{\partial \mathbf{X} / \partial \xi}{|\partial \mathbf{X} / \partial \xi|}, \quad \mathbf{n}=\kappa^{-1} \frac{\partial \mathbf{t}}{\partial s},
$$

where $\kappa$ is the curvature of the interface and $s$ is arc length.

Take the densities on the inside and outside of the contour to be $\rho_{1}$ and $\rho_{2}$, respectively. The velocity of the fluid approaching the contour from the inside and outside will be written as $\mathbf{u}_{1}$ and $\mathbf{u}_{2}$, respectively. Then the vortex sheet strength is defined as $\gamma=L\left(\mathbf{u}_{1}-\mathbf{u}_{2}\right) \cdot \mathbf{t}$. The average velocity on the interface is $\overline{\mathbf{u}}=\left(\mathbf{u}_{1}+\mathbf{u}_{2}\right) /$. We can then define a Lagrangian velocity for the interface by

$$
\widetilde{\mathbf{u}}=\overline{\mathbf{u}}+\alpha \frac{\gamma}{2 L} \mathbf{t}
$$

where $\mid d \pm$ can be chosen. This degree of freedom is possible because the contour is a curve in space and not a material object. For $\alpha=1,4$, points on the curve follow the inside and outside fluid respectively; for $\alpha=0$ points follow the average velocity.

We now write down the Euler equation on both sides of the interface,

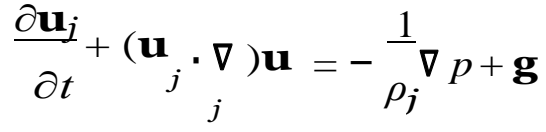

$(j=1,2)$, and work on eliminating pressure. Subtracting and re-expressing the velocity in terms of $\widetilde{\mathbf{u}}$ gives

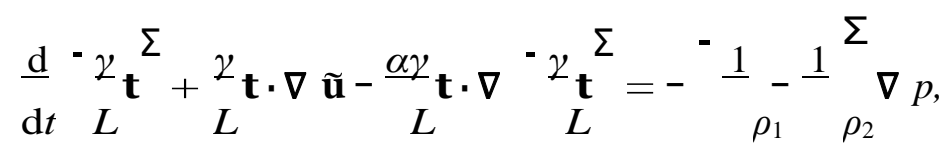

where the total derivative now represents points following $\widetilde{\mathbf{u}}$, so that

$$
\frac{\mathrm{d}}{\mathrm{d} t}=\frac{\partial}{\partial t}+\widetilde{\mathbf{u}} \cdot \boldsymbol{\nabla} .
$$

Adding the two equations in (4.3) gives

$$
2 \frac{\mathrm{d} \overline{\mathbf{u}}}{\mathrm{d} t}-\frac{\alpha \chi}{L} \mathbf{t} \cdot \nabla \overline{\mathbf{u}}+{ }_{2 L}^{\mathcal{L}} \mathbf{t} \cdot \nabla^{-\mathcal{L}_{\mathbf{t}}}{ }_{L}^{\Sigma}=--_{\rho_{2}}^{-1}{ }_{\rho_{1}}^{\Sigma} \nabla p+2 \mathbf{g} .
$$

We can now eliminate the pressure, which is continuous in the absence of surface tension, obtaining

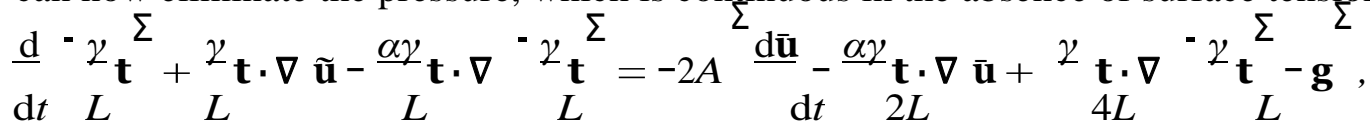

where the Atwood number is defined to be

$$
A=\frac{\rho_{1}-\rho_{2}}{\rho_{1}+\rho_{2}}
$$

(not to be confused with the constant $A$ defining the patch strength). Using the results

$$
\frac{1 \mathrm{~d} L}{L \mathrm{~d} t}=\mathbf{t} \cdot[(\mathbf{t} \cdot \boldsymbol{\nabla}) \tilde{\mathbf{u}}], \quad \mathbf{t} \cdot \boldsymbol{\nabla}=\frac{1 \partial}{L \partial \xi}, \quad \frac{1 \partial \mathbf{t}}{L \partial \xi}=\kappa \mathbf{n}, \quad \frac{1 \partial \mathbf{n}}{L \partial \xi}=-\kappa \mathbf{t}
$$


(the latter are the Frenet-Serret formulas) leads to

$$
\begin{aligned}
& \frac{\mathrm{d} \gamma}{\mathrm{d} t}-\frac{\alpha \partial}{2 L \partial \xi}-\frac{\nu^{\Sigma_{2}}}{L}=-2 A L \mathbf{t} \cdot \frac{\mathrm{d} \overline{\mathbf{u}}}{\mathrm{d} t}-\frac{\alpha \gamma \partial \overline{\mathbf{u}}}{2 L \partial \dot{\xi}} \mathbf{t}+\frac{1 \partial \partial}{8 L} \partial \xi{ }^{-\nu^{\Sigma_{2}}}-\mathbf{t} \cdot \mathbf{g} .
\end{aligned}
$$

This agrees with (2.15) in [7], although their result was for irrotational flow (see also e.g. [2]).

Setting $\alpha=0$ gives

$$
\frac{\mathrm{d} \gamma}{\mathrm{d} t}=-2 A L \mathbf{t} \cdot \frac{\mathrm{d} \overline{\mathbf{u}}}{\mathrm{d} t}+\frac{1 \partial}{8 L \partial \xi}-\nu^{\Sigma_{2}}-\mathbf{t} \cdot \mathbf{g} .
$$

This is consistent with the results of [27], which does not include gravity.

This is not a standard CD method, because the equation for $\mathrm{d} \gamma / \mathrm{d} t$ couples with that for $\mathbf{t} \cdot \mathrm{d} \overline{\mathbf{u}} / \mathrm{d} t$. This system can be turned into a Fredholm integral equation of the second kind, as shown in [7], who give a convergence proof for the equation. The derivation above carries through in two dimensions.

The Boussinesq limit gives $\mathrm{d} \gamma / \mathrm{d} t=2 A L \mathbf{t} \cdot \mathbf{g}$, which is the gravitational part of (3.6). Surface tension can also be added. The pressure is then discontinuous at the interface, with $\beta_{2} p_{1}=\kappa \rho_{0} T_{s}$, with $T_{s}$ the coefficient of surface tension. Similarly one can add a toroidal magnetic field component $B$ inside the vortex as above, giving

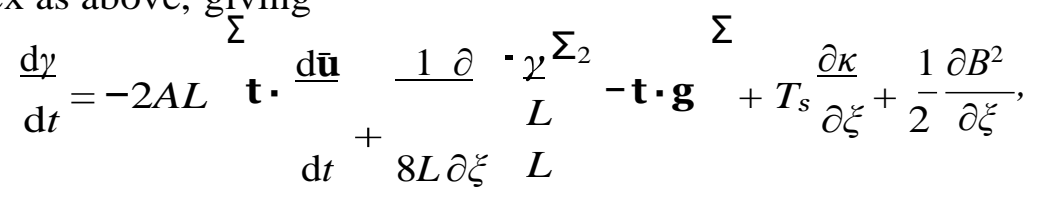

where we now take $\rho_{0}=\left(\rho_{1}+\rho_{2}\right) / 2$. Surface tension leads to a term that modifies the vortex sheet strength, proportional to the curvature of the contour, while the magnetic term is as before. Further details and numerical solutions can be found in [34].

\section{HELICAL CONTOUR DYNAMICS}

Helical geometry refers to a situation in which a function depends only on $r$ and $\varphi=\theta+s z$, the radial and helical coordinates respectively. The $\varphi$ coordinate defines a helix, with $s$ giving the pitch of the helix. The symmetry direction is given by the helical vector, $\mathbf{h}$, which is defined in cylindrical coordinates to be

$$
\mathbf{h}=h^{2}(\hat{\mathbf{z}}-s r \hat{\boldsymbol{\theta}})
$$

with $h^{2}=\left(1+s^{2} r^{2}\right)^{-1}$ (so not a unit vector). We are following the presentation of [19] for the geometry (see also [15]); the notation for velocity components differs from that in previous sections.

The goal of this section is to describe a contour dynamics approach in which this symmetry constraint is satisfied. Density is taken to be constant and there are no body forces acting. Flows with helical symmetry have velocity, vorticity and pressure fields that are invariant in the direction of $\mathbf{h}$. Helical symmetry also implies that $\mathbf{h} \cdot \boldsymbol{\nabla}=0$ for functions of $r, \varphi$ and $t$.

Upon decomposing the velocity and vorticity fields into components tangential and normal to the vector $\mathbf{h}$, for incompressible flow, we have

$$
\mathbf{u}=\mathbf{h} \times \nabla \psi+\mathbf{h} v, \quad \boldsymbol{\omega}=\mathbf{h} \times \nabla \chi+\mathbf{h} \zeta,
$$

where $\boldsymbol{\nabla}=\hat{\mathbf{r}} \partial / \partial r+\hat{\boldsymbol{\varphi}}(r h)^{-1} \partial / \partial \varphi$. The quantities $\psi$ and $v$, which depend only on $r$ and $\varphi$, have different meanings from previously. This leads to

$$
u_{r}=-\frac{1 \partial \psi}{r \partial \varphi}, \quad u_{\theta}=h^{2} \frac{\partial \psi}{\partial r}-s r v, \quad u_{z}=h^{2} v+s r^{\frac{\partial \psi}{\partial r}} .
$$

The streamfunction-vorticity relation becomes

$$
{\underset{r \partial r}{r \partial r}}_{r \partial \partial^{2}}^{\frac{1 \partial^{2}}{\partial r}}{ }^{\Sigma}+\frac{1}{r^{2}} \frac{\partial^{2} \psi}{\partial \varphi^{2}}=\omega+2 s h^{4} v
$$


The governing inviscid equations can be written as

$$
\frac{\mathrm{D} v}{\mathrm{D} t}=0, \quad \frac{\mathrm{D} \omega}{\mathrm{D} t}=2 s h^{4} \quad J(\psi, v)-s v^{\frac{\partial v^{\Sigma}}{\partial \varphi},}
$$

where $J(f, g)$ is the Jacobian in helical coordinates:

$$
J(f, g)=\frac{1^{-}}{r} \frac{\partial f \partial g}{\partial r \partial \varphi}-\frac{\partial f \partial g^{\Sigma}}{\partial \varphi \partial r} .
$$

It is clear that $v$ is materially conserved. If we take $v$ to be piecewise constant, the quantity $\omega$ is also materially conserved except on boundaries, where a vortex sheet will be induced. Hence we again write

$$
\omega=A H(f)+\Omega|\nabla f f| \delta(f), \quad v=C H(f)+v_{\infty} .
$$

Non-zero $v$ is needed outside the vortex to obtain appropriate decay at infinity. Proceeding as before shows that $A$ and $C$ are materially conserved and that $f$ is a material contour, once again. The vortex sheet strength is governed by

$$
\frac{\mathrm{D} \Omega}{\mathrm{D} t}+\frac{\Omega \mathrm{D}|\boldsymbol{\nabla} f|}{|\boldsymbol{\nabla} \boldsymbol{f}| \mathrm{D} t}=2 s h^{4} \quad C J(\psi, f)-\underline{1}_{s C^{2}} \frac{\partial \boldsymbol{f}^{\Sigma}}{2} \partial \varphi .
$$

We again introduce $\gamma=\Omega L R$, where now

and obtain

$$
L^{2}(\xi, t)=\frac{\partial R}{\partial \xi}^{\Sigma}+r \frac{\partial \Phi}{\partial \xi}^{\Sigma}
$$

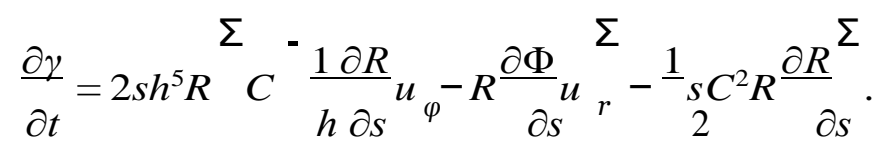

The Lagrangian advection equations for the contour $(R, \Phi)$ in helical coordinates are

$$
\frac{\partial R}{\partial t}=u_{r}(R, \Phi, t), \quad \frac{\partial \Phi}{\partial t}=\frac{1}{R h(R)} \boldsymbol{u}_{\varphi}(R, \Phi, t) .
$$

We now use the approach of [9] to obtain a contour dynamics formulation. We write the Green's function solution to (5.4) as

$$
\psi=t t\left(r, \varphi ; r^{j}, \varphi^{j}\right) F\left(r^{j}, \varphi^{j}\right) r^{j} h\left(r^{j}\right) d r^{j} d \varphi^{j},
$$

where $t t\left(r, \varphi ; r^{j}, \varphi^{j}\right)$ is an appropriate Green's function and $F(r, \varphi)=\omega+2 s h^{4} v$, where $\omega$ and $v$ are piecewise constant. The vortex sheet leads to the following contour integral for $\psi$ :

$$
\psi_{S}=t t\left(r, \varphi ; r^{j}, \varphi^{j}\right) \Omega\left(r^{j}, \varphi^{j}\right) r^{j} h\left(r^{j}\right) d s^{j},
$$

which can then be differentiated to recover the velocity $\mathbf{u}_{S}$.

To obtain the rest of the velocity field, we start with Green's theorem in the form

$$
{ }_{V} \quad \frac{\partial Q}{\partial r^{j}}-\frac{1 \partial P}{r^{j} h^{j} \partial \varphi^{j}} r^{j} h^{j} d r^{j} d \varphi^{j}={ }_{C}\left(P d r^{j}+r^{j} h^{j} Q d \varphi^{j}\right),
$$

where $h^{j}=h\left(r^{j}\right)$ and so on. On defining new functions using

we can obtain

$$
r h \frac{\partial P}{\partial \varphi^{j}}=r^{j} h^{j} \frac{\partial t t}{\partial \varphi}, \quad \frac{\partial Q}{\partial r^{j}}=\frac{1}{h^{j 4}} \frac{\partial S}{\partial r^{j}}=\frac{\partial t t}{\partial r},
$$

$$
\frac{\partial \psi_{H}}{\partial \varphi}=-\frac{1}{r h}^{\mathbf{I}} P F^{j} d r^{j}, \quad \frac{\partial \psi_{H}}{\partial r}=A \quad Q r^{j} h^{j} d \varphi^{j}+2 s C^{\mathbf{I}} S r^{j} h^{j} d \varphi^{j} .
$$


These can then be substituted into (5.3).

Obtaining the Green's function is more challenging than for the previous cases. Taking the source point to have coordinates $r_{0}$ and $\varphi_{0}=0$ (general $\varphi_{0}$ follows immediately), Fourier transforming in the azimuthal direction leads to a decomposition of $t t$ into azimuthal modes of the form

$$
t t\left(r, \varphi ; r_{0}, 0\right)=\sum_{m}^{\boldsymbol{t}_{m}\left(r ; r_{0}\right) e^{i m \varphi},}
$$

while (5.4) leads to the following set of ordinary differential equations

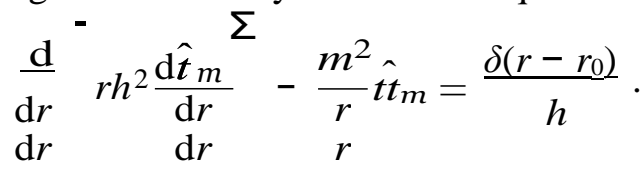

The modes with non-zero $m$ satisfy

$$
\frac{r}{1+s^{2} r^{2}}{ }^{\hat{j}} t_{m}+\frac{1-s^{2} r^{2}}{\left(1+s^{2} r^{2}\right)^{2}} \hat{\imath} t_{m}^{j}-{\frac{m^{2}}{r}}_{t t_{m}}=\frac{\delta\left(r-r_{0}\right)}{h} .
$$

It can be shown that $\hat{\boldsymbol{t}}_{m}$ takes the form

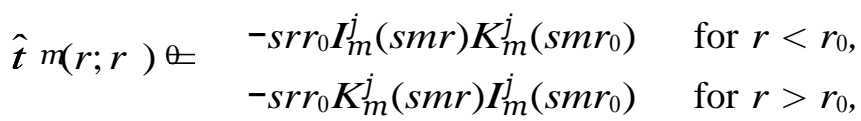

where $I_{m}$ and $K_{m}$ are modified Bessel functions of the first and second kind of order $m$. The solution for the axisymmetric mode can be obtained in closed form following [19]. Further details can be found in [35].

Swirl along the vortex axis is naturally included in the form of $v$. Buoyancy cannot be introduced in a helically symmetric form that is consistent with a CD formulation. Gravity is a constant so its component in the $x$ and $y$ directions cannot be helically symmetric, while its $z$-component does not allow a CD formulation (as in two dimensions). However, a magnetic field along the vortex can be introduced. The Euler and induction equation in helical geometry can be found in [30]. The magnetic field then takes the form $\mathrm{Ch}^{-2} \mathbf{h}$, which is consistent with the two-dimensional and axisymmetric forms ( $C \hat{\mathbf{z}}$ and $C r \hat{\boldsymbol{\theta}}$ respectively).

\section{CONCLUSIONS}

To compute the motion, one needs an inversion relation linking vortex patch and sheet strength to velocity, (2.14), (2.21) and (2.23), and (5.13) and (5.16) in the two-dimensional, axisymmetric and helical cases respectively; an advection equation for the material points on the boundary (2.11); and finally evolution equations for the vortex sheet strength, (3.6) and (3.9) in the two-dimensional and axisymmetric Boussinesq cases, and (5.10) in the helical case. In the non-Boussinesq case, one solves (4.12) coupled to an equation for $\mathrm{d} \overline{\mathbf{u}} / \mathrm{d} t$ obtained by taking time derivatives of the velocity field evaluated on the interface.

It is clear that all of the derivations above can be generalized to multiple contours. The notation becomes more complicated, and the velocity is given by integrals around all of the contours. Similarly, simple geometries such as half-plane, right-angle corners, and inside and outside circles can be handled in planar geometry by using the method of images. Periodic and general orientable two-dimensional surfaces and multiply-connected geometries can be tackled using appropriate Green's functions $[8,9]$. A half-space with a horizontal boundary can similarly be considered in axisymmetric geometry.

One message is that irrotational flow is not needed to obtain equations for vortex sheet motion even in the presence of density differences: the component of the momentum equation tangent to the sheet suffices and the unsteady Bernoulli equation is not needed.

As pointed out by [23], extensions of contour dynamics to compressible situations are problematic because the velocity is no longer solenoidal. The case of a fluid with background stratification similarly suffers because vorticity is generated everywhere in the bulk by baroclinic torques. Asymptotic approaches have been attempted [33], but they are limited to small times. The approach 
of [10] may show promise, but has so far only been developed in two dimensions and for small perturbations to boundaries.

A final conclusion is that when contour dynamics can be extended to include additional physics, the outcome is a coupled vortex patch-vortex sheet system, and numerical simulations of the evolution of vortex sheets are known to be complicated by the existence of singularities.

\section{ACKNOWLEDGMENTS}

Part of this research was supported by NSF Award CBET-1706934. Support from Collaborative Research Project 2017 and 2018, Institute of Fluid Science, Tohoku University, Project Codes J16R004 and J17R004 is also acknowledged.

\section{REFERENCES}

Books:

1. Alekseenko, S. V., Kuibin, P. A., and Okulov, V. L. Theory of Concentrated Vortices: An Introduction, Berlin: Springer, 2007.

2. Pozrikidis, C. Introduction to Theoretical and Computational Fluid Dynamics, 2nd ed., New York: Oxford University Press, 2011.

3. Newton, P. K., The N-Vortex Problem, New York: Springer, 2001.

4. Saffman, P. G., Vortex Dynamics, Cambridge: Cambridge University Press, 1992.

5. Vallis, G. K., Atmospheric and Oceanic Fluid Dynamics, 2nd ed., Cambridge: Cambridge University Press, 2017.

6. Wu, J.-Z., Ma H.-Y., and Zhou, M.-D. Vorticity and Vortex Dynamics, Berlin: Springer, 2006.

Journal Papers:

7. Baker, G. R., Meiron, D. I., and Orszag, S. A., Generalized vortex methods for free-surface flow problems, J. Fluid Mech., 1982, vol. 123, pp. 477-501.

8. Crowdy, D. G. and Surana, A. Contour dynamics in complex domains, J. Fluid Mech., 2007, vol. 593, pp. 235-254.

9. Dritschel, D. G. Contour dynamics and contour surgery: Numerical algorithms for extended highresolution modelling of vortex dynamics in two-dimensional, inviscid, incompressible flows, Comput. Phys. Rep.J. Comp. Phys., 1989, vol. 10, pp. 79-146.

10. Gorshkov, K. A., Ostrovsky, L. A., and Soustova, I. A., Perturbation theory for Rankine vortices, J. Fluid Mech., 2000, vol. 404, pp. 1-25.

11. Hattori, Y. and Llewellyn Smith, S. G., Motion of axisymmetric magnetic eddies with swirl, Procedia IUTAM, 2013, vol. 7, pp. 243-250.

12. Hattori, Y. and Moffatt, H. K., Evolution of toroidal magnetic eddies in an ideal fluid, J. Fluid Mech., 2006, vol. 558, pp. 253-279.

13. Helmholtz, H., Uber Integrale der hydrodynamischen Gleichungen welche den Wirbelbewegungen entsprechen, Crelles J., 1858, vol. 55, pp. 25-55.

14. Krasny, R. Desingularization of periodic vortex sheet roll-up. J. Comp. Phys., 1986, vol. 65, pp. $292-313$.

15.Landman, M. J., On the generation of helical waves in circular pipe flow. Phys. Fluids A, vol. 2, pp. 738747.

16. Llewellyn Smith, S. G., How do singularities move in potential flow?, Physica D, 2011, vol. 240, pp. 16441651.

17. Llewellyn Smith, S. G. and Hattori, Y., Axisymmetric magnetic vortices with swirl, Commun. Nonlinear Sci. Numer. Simulat., 2012, vol. 17, pp. 2101-2107.

18. Llewellyn Smith, S. G. and Tobias, S. M., Vortex dynamos, J. Fluid Mech., 2004, vol. 498, pp. 1-21.

19.Lucas, D. and Dritschel, D. G., A family of helically symmetric vortex equilibria, J. Fluid Mech., 2009, vol. 634, pp. 245-268.

20. Meleshko, V. V. and Aref, H., A bibliography of vortex dynamics 1858-1956, Adv. Appl. Mech., 2007, vol. 451, pp. 197-292.

21. Norbury, J. A family of steady vortex rings, J. Fluid Mech., 1973, vol. 57, pp. 417-431.

22.Pozrikidis, C., The nonlinear instability of Hill's vortex, J. Fluid Mech., 1986, vol. 168, pp. $337-367$.

23.Pullin, D. I. Contour dynamics methods, Annu. Rev. Fluid Mech., 1992, vol. 24, pp. 89-115.

24. Pullin, D. I. and Jacobs, P. A., Inviscid evolution of stretched vortex arrays. J. Fluid Mech., 1986, vol. 171, pp. 377-406.

25. Riley, N. The fascination of vortex rings, Appl. Sci. Res., vol. 59, pp. 169-189.

26. Shariff, K., Leonard, A., and Ferziger, J., A contour dynamics algorithm for axisymmetric flow, J. Comp. Phys., 2008, vol. 227, pp. 9044-9062.

27. Shin, S., Sohn, S.-I., and Hwang, W. Vortex simulations of the Kelvin-Helmholtz instability with surface tension in density-stratified flows, Eur. J. Mech. B/Fluids, 2018, vol. 67, pp. 168-177. 
28. Tryggvason, G., Numerical simulations of the Rayleigh-Taylor instability. J. Comp. Phys., 1988, vol. 75, pp. 253-282.

29. Wu, H. M., Overman II, E. A., and Zabusky, N. J., Steady-state solutions of the Euler equations in two dimensions: rotating and translating V-states with limiting cases. I. Numerical algorithms and results, J. Comp. Phys., 1984, vol. 53, pp. 42-71.

30. Zabielski, L. and Mestel, A. J., Kinematic dynamo action in a helical pipe, J. Fluid Mech., 2005, vol. 535, pp. 347-367.

31. Zabusky, N. J., Hughes, M. H., and Roberts, K. V., Contour dynamics for the Euler equations in two dimensions, J. Comp. Phys., 1979, vol. 30, pp. 96-106.

Technical Reports:

32. Shariff, K., Leonard, A., and Ferziger, J., Dynamics of a Class of Vortex Rings, NASA, 102257. Dissertations and Theses:

33. Griffiths, R. M., Interaction of Vorticity and Internal Gravity Waves, PhD Thesis, Cambridge University, Cambridge, 1999.

Preprints:

34. Ching, C. and Llewellyn Smith, S. G. An axisymmetric vortex ring in the non-Boussinesq regime, Under preparation. 2018.

35. Chu, T., Llewellyn Smith, S. G., and Ching, C. Helical contour dynamics. Under preparation. 2018. 